Published in final edited form as:

Conf Proc IEEE Eng Med Biol Soc. 2012 August ; 2012: 314-317. doi:10.1109/EMBC.2012.6345932.

\title{
Contralaterally Controlled Functional Electrical Stimulation for Stroke Rehabilitation
}

\author{
Jayme S. Knutson, \\ Cleveland Functional Electrical Stimulation Center and the Department of Physical Medicine and \\ Rehabilitation at MetroHealth Medical Center and the School of Medicine at Case Western \\ Reserve University, Cleveland OH 44106 USA, phone: 216-778-8364; fax: 216-778-5560 \\ Mary Y. Harley, \\ Department of Physical Medicine and Rehabilitation at MetroHealth Medical Center, Cleveland \\ OH 44109 USA
}

Terri Z. Hisel,

Department of Physical Medicine and Rehabilitation at MetroHealth Medical Center, Cleveland $\mathrm{OH} 44109$ USA

Nathaniel S. Makowski,

Cleveland Functional Electrical Stimulation Center and the Department of Biomedical Engineering at Case Western Reserve University, Cleveland OH 44106 USA

Michael J. Fu, and

Cleveland Functional Electrical Stimulation Center and the Louis Stokes Cleveland VA Medical Center, Cleveland $\mathrm{OH} 44106$ USA

\section{John Chae}

Cleveland Functional Electrical Stimulation Center and the Department of Physical Medicine and Rehabilitation at MetroHealth Medical Center and the School of Medicine at Case Western Reserve University, Cleveland OH 44106 USA

Jayme S. Knutson: jsk12@case.edu; Mary Y. Harley: mharley@metrohealth.org; Terri Z. Hisel: thisel@metrohealth.org; Nathaniel S. Makowski:nhm6@case.edu; Michael J. Fu: mjf24@case.edu; John Chae: jchae@metrohealth.org

\section{Abstract}

Contralaterally controlled functional electrical stimulation (CCFES) is an innovative method of delivering neuromuscular electrical stimulation for rehabilitation of paretic limbs after stroke. It is being studied to evaluate its efficacy in improving recovery of arm and hand function and ankle dorsiflexion in chronic and subacute stroke patients. The initial studies provide preliminary evidence supporting the efficacy of CCFES.

\section{Introduction}

Stroke is the leading cause of adult disability in the United States, with about 800,000 strokes occurring each year. Many stroke survivors have chronic hemiplegia that affects motor function in both the upper and lower extremity. In the upper extremity, paretic finger extensors and spastic forearm flexors make it difficult to open and use the hand. In the lower extremity, paretic ankle dorsiflexors cause the foot to drag during the swing phase of gait, resulting in unsafe and inefficient ambulation or non-ambulation.

Contralaterally controlled functional electrical stimulation (CCFES) is a new therapy designed to improve the recovery of paretic limbs after stroke. CCFES uses a control signal from the non-paretic side of the body to regulate the intensity of electrical stimulation delivered to the paretic muscles of the homologous limb on the opposite side of the body [1]. 
For example, with Hand CCFES, the stimulation intensity (e.g., current pulsewidth) delivered to paretic hand extensors is proportional to the degree of finger extension of the unaffected hand. An instrumented glove worn on the unaffected hand supplies the control signal to the stimulator (Fig. 1). CCFES treatment consists of: 1) self-administered repetitive stimulated movement exercises at home, and 2) therapist-guided task practice at the rehabilitation clinic.

The purpose of this article is to introduce CCFES to the neural and rehabilitation engineering community, review the rationale for CCFES, summarize the studies of CCFES to date, and present preliminary data on a version of CCFES that includes stimulation of both elbow and hand extensors.

\section{Rationale for Contralaterally Controlled Functional Electrical Stimulation}

The CCFES paradigm incorporates several rehabilitation principles that are important for motor relearning. First, CCFES puts the brain back in control of the affected limb by giving the patient direct proportional control of the stimulation intensity. This capitalizes on the principle of intention-driven movement, maximizing the degree of synchronization between motor intention (central neural activity) and stimulated motor response (peripheral neural activity). This repetitive synchronizing of neural activity along central and peripheral motor and sensory neural pathways may promote synaptic remodeling and neural reorganization leading to improved central control of the impaired limb [2-4]. With CCFES, the patient not only controls when the stimulation turns on (as is also the case with EMG-triggered or switch-triggered stimulation), but also controls the duration and intensity of stimulation and therefore the resultant movement.

Second, CCFES incorporates bilateral movement principles by having the contralateral unaffected limb control stimulation and consequent movement of the affected limb. Because users are instructed to try to move their affected limb in concert with the unaffected limb, the treatment may encourage bilateral cortical activity. Bilateral symmetric movement exercise has been shown to reduce upper limb impairment [5], and the CCFES technique may increase corticospinal excitability of the stimulated muscles [6].

Third, CCFES creates a strong perception of restored motor control because the stimulated limb moves according to the patient's motor intention. Creating the illusion of restored motor control beyond the current capabilities of the affected limb may drive neuroplastic change and motor recovery [7]. Mental practice, mirror therapy, and interactive media training (e.g., virtual reality) are based on this principle.

Fourth, because the user controls the timing and degree of stimulated hand opening in a way that does not interfere with the fluidity of task practice, the CCFES system enables the patient to practice goal-oriented functional tasks and/or motor control tasks (Fig. 2). Active (self-initiated), repetitive, goal-oriented, skill-requiring task practice is critical in driving functional reorganization of cortical maps and facilitating motor recovery after stroke $[2,8]$.

Furthermore, CCFES treatment does not require any residual hand or ankle movement and can be delivered with a high dose because the device is portable and the treatment is simple enough to be self-administered at home.

\section{CCFES Device}

The CCFES device consists of a stimulator, an instrumented glove (for the hand application) or sock (for the ankle application), and surface electrodes (Fig. 3). The stimulator can deliver up to 7 monopolar channels of biphasic current. Each channel can be individually 
programmed to send and modulate the stimulation intensity in accordance with input(s) from external transducers or automatically according to a fixed duty cycle (i.e., cyclic

stimulation). Customized patterns of stimulation can be programmed by specifying for each stimulus channel a stimulus intensity (i.e., pulse duration) profile, which defines how the stimulation increases as a function of the input signal from the glove or sock, or alternatively, as a function of time (for cyclic stimulation). Sound and light cues can be programmed to prompt the user to open their hands (Hand CCFES) or dorsiflex their ankles (Ankle CCFES) repeatedly over an exercise session of selectable duration. An internal memory stores usage statistics. Matlab Simulink ${ }^{\circledR}$ is used to program the stimulator.

The command glove (for Hand CCFES) is an off-the-shelf bike glove with an assembly of 3 bend sensors (Images SI Inc., Staten Island, NY) sewn into cloth sheaths affixed with Velcro to the dorsal aspect of digits II-III-IV. The sock (for Ankle CCFES) has a single bend sensor attached across the dorsal aspect of the ankle. The electrodes are commercially available pre-gelled surface electrodes (PALS, Axelgaard Manufacturing Co., Fallbrook, CA) of various sizes selected to best target small and large muscles in the forearm, hand, or leg.

\section{Recovery of Hand Opening with Contralaterally Controlled FES}

Two pilot case series studies [1,9] and an early-phase randomized controlled trial (RCT) [10] have been conducted to estimate the efficacy of CCFES to reduce hand impairment and improve hand function. The first two pilot studies included a total of 6 participants with chronic (> 6 months) hemiplegia; the RCT included 21 subacute ( $<6$ months) patients. The treatment regimen in all of these studies was similar: a 55-min session of cued repetitive CCFES-mediated hand opening exercise twice daily at home plus a 90-min session of functional task practice in the lab twice weekly. The treatment period was 6 weeks in the first pilot study, 12 weeks in the second pilot study, and 6 weeks in the RCT.

All 6 participants in the two pilot studies experienced reductions in at least one measure of hand impairment by end-of-treatment [1,9]. All 6 had increases in Box and Blocks score (number of blocks picked up and released over a barrier in 1 minute) ranging from 3 to 15 more blocks; 5 had modest (13\%) to marked (87\%) reductions in finger movement tracking error (a measure of motor control); 4 had modest $\left(8^{\circ}\right)$ to marked $\left(101^{\circ}\right)$ increases in maximum active finger extension angle; and all 3 who were tested with the Fugl-Meyer assessment (a measure of motor impairment with a maximum score of 66) had improvements ranging from 7 to 15 points. Participants who had 12 weeks of treatment showed clinically significant gains between the 6-wk and 12-wk time points, suggesting the longer treatment duration may bring greater benefits [9].

The early-phase RCT compared CCFES to cyclic neuromuscular electrical stimulation (NMES), a commonly used method of repeatedly stimulating the paretic limb automatically without any linkage to motor intention or voluntary contraction from either limb [10]. For each of the measures of hand impairment and function used in the study, the CCFES group experienced greater improvement on average than the cyclic NMES group, providing evidence that CCFES therapy's key ingredients (pairing motor intention to motor response, bilateral symmetric movement, and stimulation-assisted practice of goal-oriented tasks) have promise for treating hemiplegia. Although the treatment effect did not reach statistical significance, the outcome measure showing the largest treatment effect was maximum voluntary finger extension, with the CCFES group having $28^{\circ}\left[95 \% \mathrm{CI}:-4^{\circ}\right.$ to $60^{\circ}$ ] more finger extension on average across the post-treatment period than the cyclic NMES group.

In addition to these studies, a large RCT that plans to enroll 102 chronic patients is presently ongoing and aims to estimate the effects of baseline impairment and treatment duration on 
outcome and will evaluate the persistence of the treatment effect over a 6-month follow-up period.

All the participants in these studies were able to put on and use the CCFES system independently at home approximately $2 \mathrm{hrs}$ per day as instructed. In general, the study participants' responses to Hand CCFES have been very enthusiastic. Of 32 CCFES participants who were given a questionnaire at the end of their treatment, 29 (91\%) agreed or strongly agreed with the statement, "The exercises and therapy associated with this study improved my hand function."

\section{Arm + Hand CCFES for Recovery of Simultaneous Reach and Hand Opening}

Many stroke patients lose not only the ability to open their paretic hand, but also cannot extend their elbow and therefore have a very limited workspace. Arm+Hand CCFES is intended to improve simultaneous reaching and hand opening. Arm+Hand CCFES adds elbow extensor stimulation to the Hand CCFES system. A cuff with a bend sensor is worn around the elbow of the unaffected arm (Fig. 4). The sensor is connected to the stimulator and its input modulates the intensity of stimulation delivered to the triceps muscle of the paretic arm such that greater extension of the unaffected elbow produces greater stimulation of the affected triceps. Biceps stimulation can be added for patients who cannot flex their elbow.

A pilot case series study of Arm+Hand CCFES is currently enrolling stroke patients with chronic (> 6 months) hemiplegia who are unable to reach forward and open their hand fully at the same time. Like Hand CCFES, Arm+Hand CCFES treatment consists of home-based exercise (cued "reach-and-open") and lab-based CCFES-mediated practice of reaching tasks and hand tasks. During the home CCFES-mediated "reach-and-open" exercises, the participants rest both forearms in mobile arm supports (MAS) (Fig. 4) to prevent shoulder fatigue and involuntary activation of arm and forearm flexors [11]. The treatment lasts 12 weeks.

The first two participants in this study had baseline Fugl-Meyer scores (a measure of upper limb motor impairment) of 13 and 35, respectively, out of a maximum score of 66. Both participants had no functional hand opening. Table 1 shows the changes from baseline on several measures of arm and hand impairment for each participant. Measurements of voluntary elbow extension were made with a camera-based LED-marker tracking system. Voluntary hand opening was measured with a sensor attached to the hand that measures the distance between the tips of the fingers and the tip of the thumb [12]. Subjects 1 and 2 had improvements in Fugl-Meyer score, elbow extension, elbow tracking, and elbow extension during attempts to open the hand. Neither of the participants had improvements in voluntary hand opening regardless of whether they were attempting to reach simultaneously. Both subjects were able to don the electrodes and glove independently, but needed assistance from a caregiver to slide the elbow cuff onto their non-paretic arm. We recently developed wireless glove and elbow cuff prototypes that are expected to improve ease of use. Additional participants are being enrolled to further investigate the feasibility of Arm+Hand CCFES to improve simultaneous reach and hand opening.

\section{Recovery of Ankle Dorsiflexion with Contralaterally Controlled FES}

A pilot study of 3 stroke patients with chronic (> 6 months) lower extremity hemiplegia was conducted to evaluate the feasibility of CCFES to improve ankle dorsiflexion [13].

Electrodes were positioned over the peroneal nerve and the tibialis anterior to elicit strong 
ankle dorsiflexion (Fig. 5). The 6-wk treatment consisted of a 55-min session of cued repetitive CCFES-mediated ankle dorsiflexion exercise self-administered twice daily at home plus a twice weekly lab session in which participants practiced a 15-min ankle movement tracking task. The tracking task required the participants to make CCFESmediated ankle movements to control the vertical displacement of a cursor on a computer screen and thereby trace a target path scrolling across the display.

Two of the three participants experienced appreciable improvements in maximum active ankle dorsiflexion $\left(\Delta=13^{\circ}\right.$ and $\left.17^{\circ}\right)$, ankle movement tracking error $(\Delta=-57 \%$ and $-57 \%)$, and lower limb Fugl-Meyer score ( $\Delta=4$ and 5 points) [13]. The third participant had no notable changes in these measures. An early-phase RCT comparing CCFES to cyclic NMES is presently being conducted to estimate the efficacy of CCFES to reduce ankle impairment and improve gait.

\section{Future Directions for Contralaterally Controlled FES}

Increasing the amount of time patients use CCFES in the context of skill-requiring motor control tasks may further improve outcomes [14]. The previous CCFES studies included $\sim 2$ hrs/day of self-administered repetitive CCFES-mediated movement exercise in response to regularly paced auditory cues, which the participants performed at home. Although compliance with that regimen has been generally high (in the context of a research study), the repetitive task is not goal-oriented and can be performed successfully even when attention drifts away from the task. Therefore, we plan to develop motor control tasks/games in interactive media environments that can be used in conjunction with CCFES in order to maximize cognitive engagement during the CCFES-mediated exercise portion of the treatment. Virtual reality (VR) tasks developed and tested in the laboratory will be made into a portable motor-control VR module to be used in conjunction with CCFES by stroke patients at home.

CCFES+VR therapy seeks to combine the advantages of CCFES and VR therapies: intention-driven movement, bilateral neural activity, repetitive movement, maximum subject attentiveness, and tasks that motivate goal-driven motor skills. Figure 6 illustrates the labbased CCFES+VR system to be developed. Sensors and/or motion capture cameras will be used to detect movement of the paretic hand which will be represented on a computer screen in various ways, depending on the task/game. Several motor control task/games having adjustable difficulty level and means of scoring performance will be developed to encourage patients to improve their hand motor control and function. The VR tasks/games will be developed through an iterative process involving input from physiatrists, neuroscientists, therapists, engineers, computer scientists, and patients. After the tasks/games have undergone prototype testing, a pilot RCT comparing VR+CCFES and CCFES will be conducted.

\section{Acknowledgments}

The CCFES stimulators were developed by the Technical Development Laboratory of the Cleveland FES Center, Case Western Reserve University, Cleveland, OH 44106 USA.

Research supported by the National Institutes of Health National Institute of Child Health and Human Development grants R21HD054749, R01HD059814, R21HD06159, the National Center for Research Resources grants K12RR023264, KL2RR024990, the State of Ohio Biomedical Research and Technology Transfer grant BRTT03-10, and the Department of Veterans Affairs Cleveland Functional Electrical Stimulation Center. 


\section{References}

1. Knutson JS, Harley MY, Hisel TZ, Chae J. Improving hand function in stroke survivors: a pilot study of contralaterally controlled functional electric stimulation in chronic hemiplegia. Arch Phys Med Rehabil. Apr.2007 88:513-20. [PubMed: 17398254]

2. Nudo RJ, Wise BM, SiFuentes F, Milliken GW. Neural substrates for the effects of rehabilitative training on motor recovery after ischemic infarct. Science. Jun 21.1996 272:1791-4. [PubMed: $8650578]$

3. Rushton DN. Functional electrical stimulation and rehabilitation--an hypothesis. Med Eng Phys. Jan.2003 25:75-8. [PubMed: 12485788]

4. Kimberley TJ, Lewis SM, Auerbach EJ, Dorsey LL, Lojovich JM, Carey JR. Electrical stimulation driving functional improvements and cortical changes in subjects with stroke. Exp Brain Res. Feb. 2004 154:450-60. [PubMed: 14618287]

5. Luft AR, McCombe-Waller S, Whitall J, Forrester LW, Macko R, Sorkin JD, Schulz JB, Goldberg AP, Hanley DF. Repetitive bilateral arm training and motor cortex activation in chronic stroke: a randomized controlled trial. JAMA. Oct 20.2004 292:1853-61. [PubMed: 15494583]

6. Schmidt MW, Hinder MR, Summers JJ, Garry MI. Long-lasting contralateral motor cortex excitability is increased by unilateral hand movement that triggers electrical stimulation of opposite homologous muscles. Neurorehabil Neural Repair. Jul-Aug;2011 25:521-30. [PubMed: 21436392]

7. Yavuzer G, Selles R, Sezer N, Sutbeyaz S, Bussmann JB, Koseoglu F, Atay MB, Stam HJ. Mirror therapy improves hand function in subacute stroke: a randomized controlled trial. Arch Phys Med Rehabil. Mar.2008 89:393-8. [PubMed: 18295613]

8. Taub E, Uswatte G, Pidikiti R. Constraint-Induced Movement Therapy: a new family of techniques with broad application to physical rehabilitation--a clinical review. J Rehabil Res Dev. Jul.1999 36:237-51. [PubMed: 10659807]

9. Knutson JS, Hisel TZ, Harley MY, Chae J. A novel functional electrical stimulation treatment for recovery of hand function in hemiplegia: 12-week pilot study. Neurorehabil Neural Repair. Jan. 2009 23:17-25. [PubMed: 18812432]

10. Knutson JS, Harley MY, Hisel TZ, Hogan SD, Maloney MM, Chae J. Contralaterally controlled functional electrical stimulation for upper extremity hemiplegia: an early-phase randomized clinical trial in subacute stroke patients. Neurorehabil Neural Repair. Aug 29.2011 23:239-246. 2012. [PubMed: 21875892]

11. Miller LC, Dewald JP. Involuntary paretic wrist/finger flexion forces and EMG increase with shoulder abduction load in individuals with chronic stroke. Clin Neurophysiol. Feb 22.2012

12. Memberg WD, Crago PE. An analysis of the input-output properties of neuroprosthetic hand grasps. J Rehabil Res Dev. Jan-Feb;2000 37:11-21. [PubMed: 10847568]

13. Knutson JS, Chae J. A novel neuromuscular electrical stimulation treatment for recovery of ankle dorsiflexion in chronic hemiplegia: a case series pilot study. Am J Phys Med Rehabil. Aug.2010 89:672-82. [PubMed: 20531158]

14. Lang CE, Macdonald JR, Reisman DS, Boyd L, Jacobson Kimberley T, Schindler-Ivens SM, Hornby TG, Ross SA, Scheets PL. Observation of amounts of movement practice provided during stroke rehabilitation. Arch Phys Med Rehabil. Oct.2009 90:1692-8. [PubMed: 19801058] 


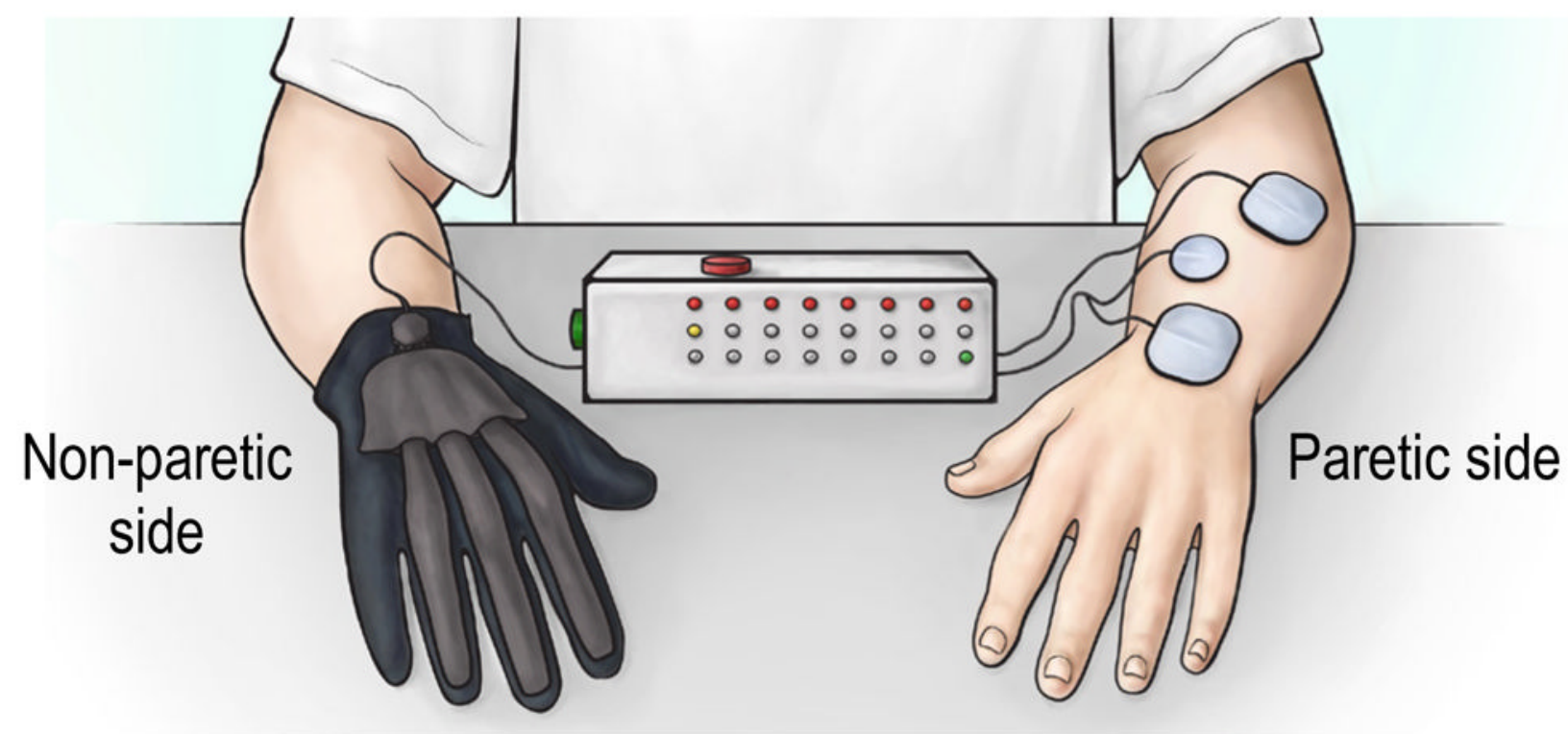

Figure 1.

Contralaterally controlled FES for improving recovery of hand opening. (92011 Cleveland FES Center. 


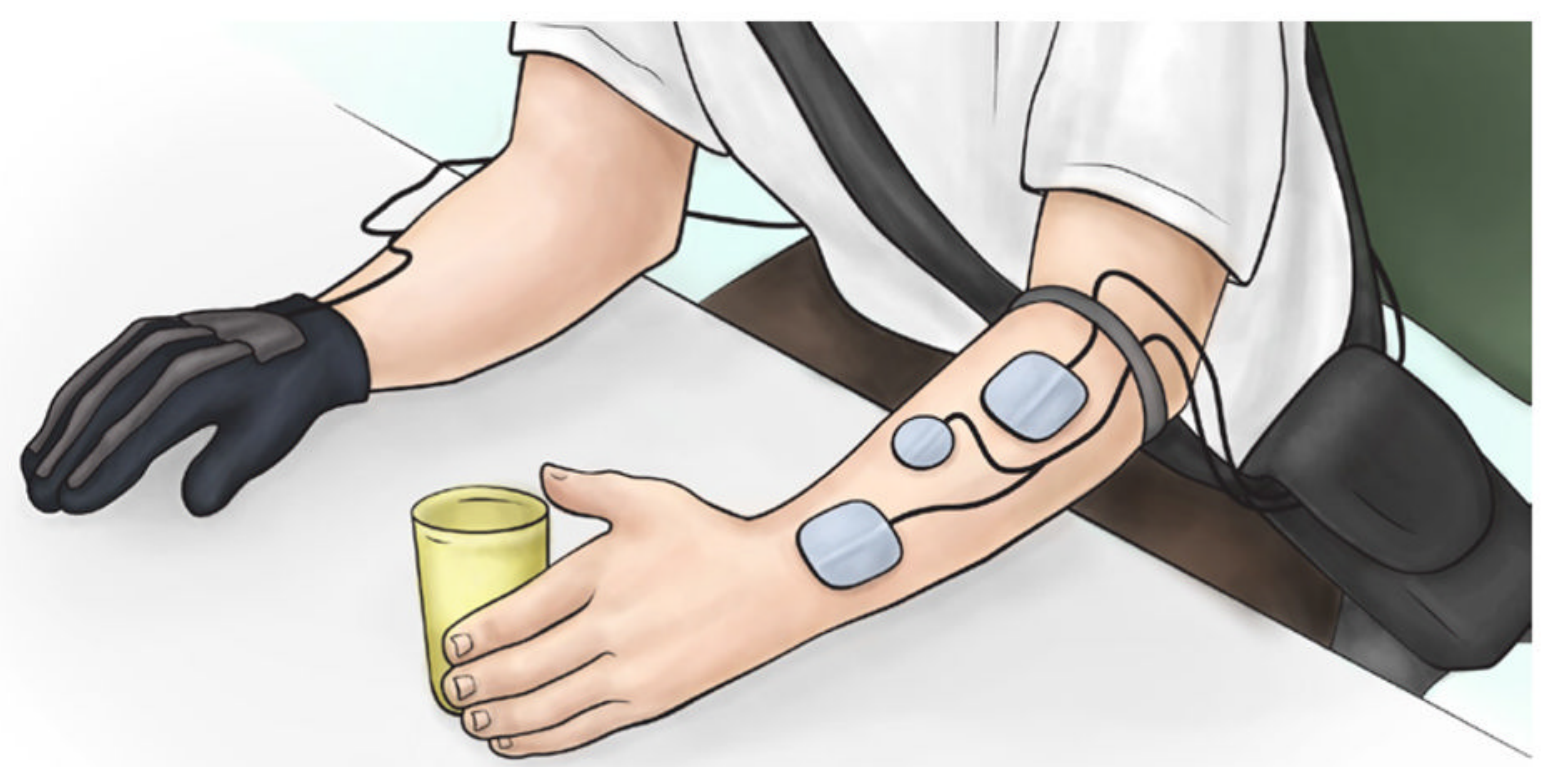

Figure 2.

CCFES makes it possible to practice functional tasks. @2011 Cleveland FES Center 


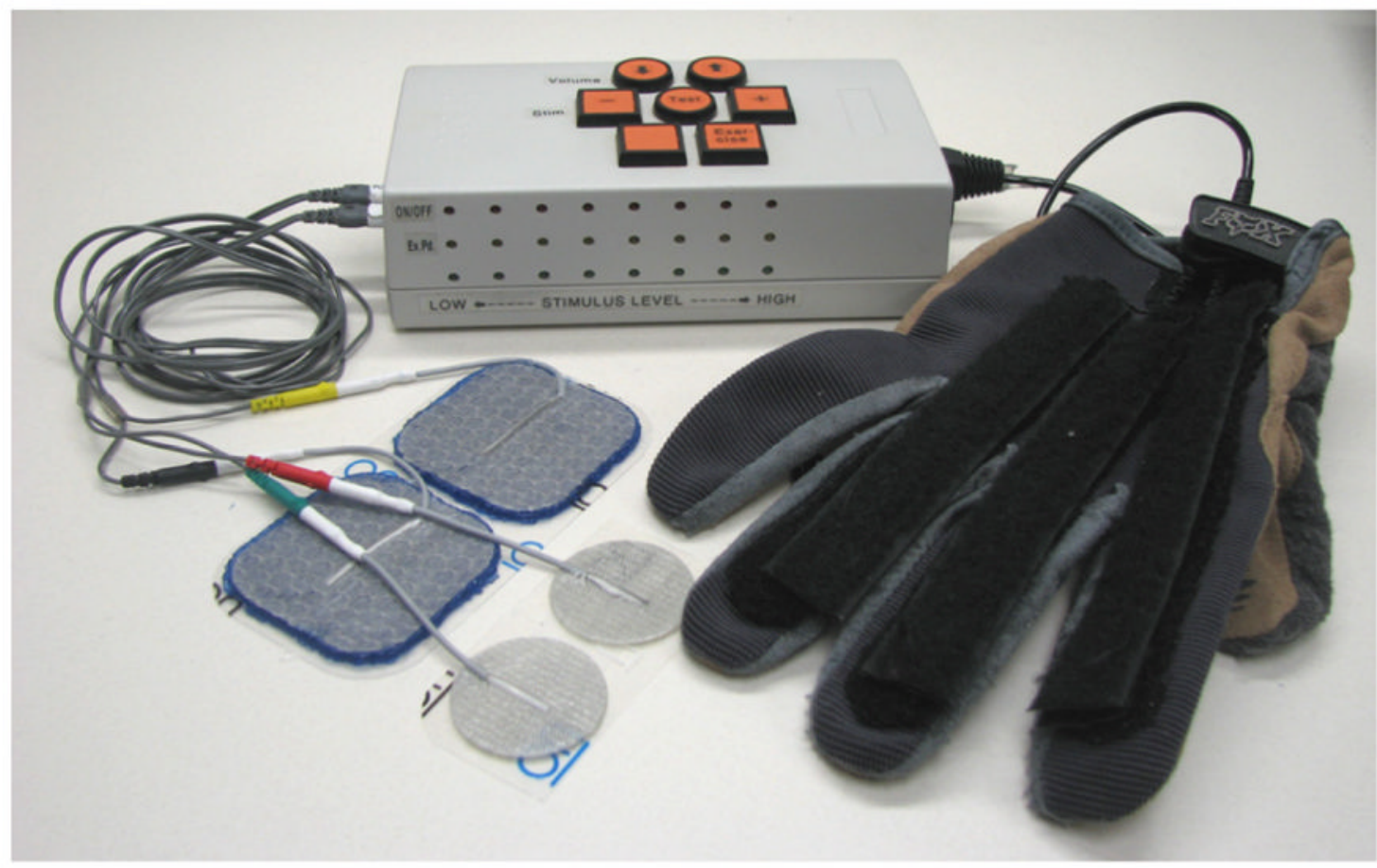

Figure 3.

CCFES stimulator, command glove, and electrodes @2012 Cleveland FES Center 


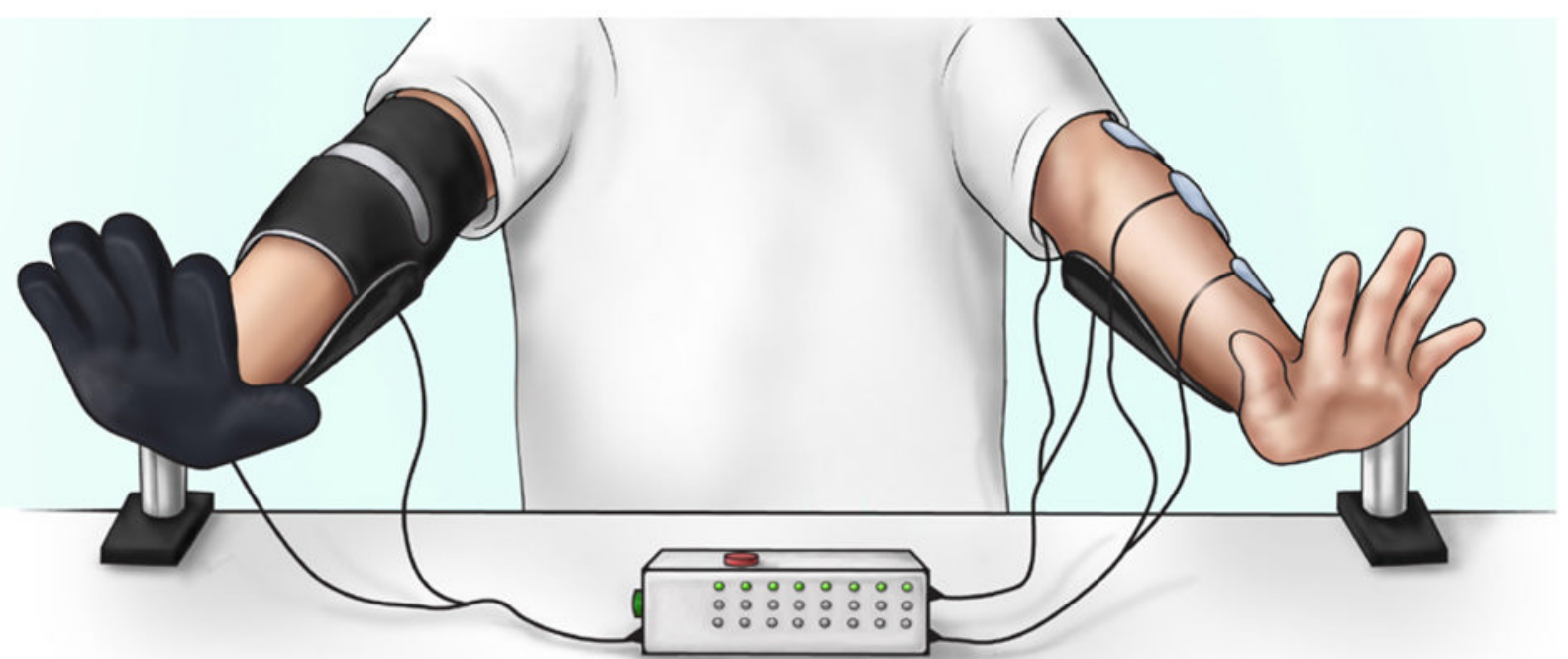

Figure 4.

Arm + Hand CCFES for improving recovery of simultaneous reach and open @2011 Cleveland FES Center 


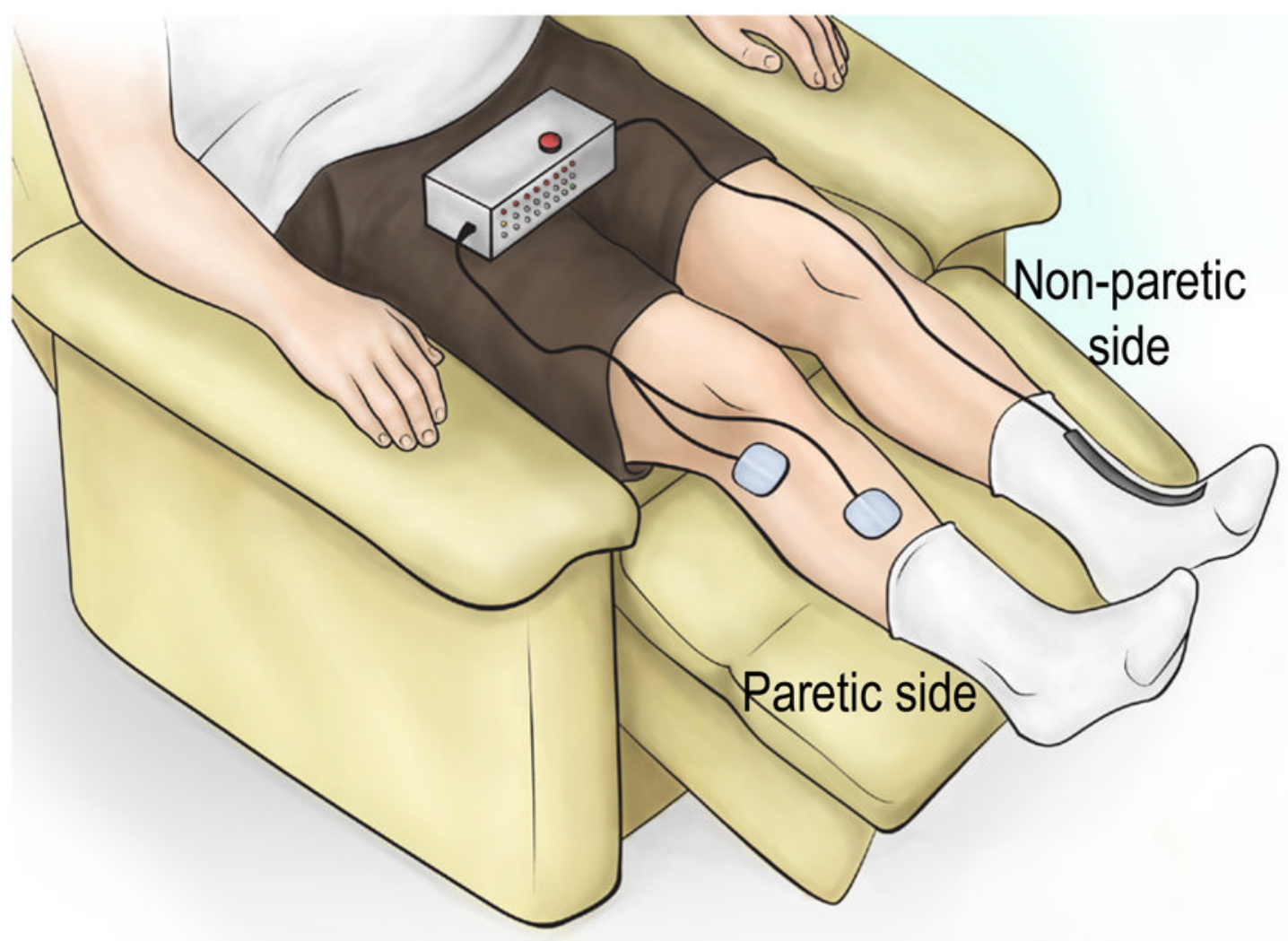

Figure 5.

Contralaterally controlled FES for improving recovery of ankle dorsiflexion @2011 Cleveland FES Center 


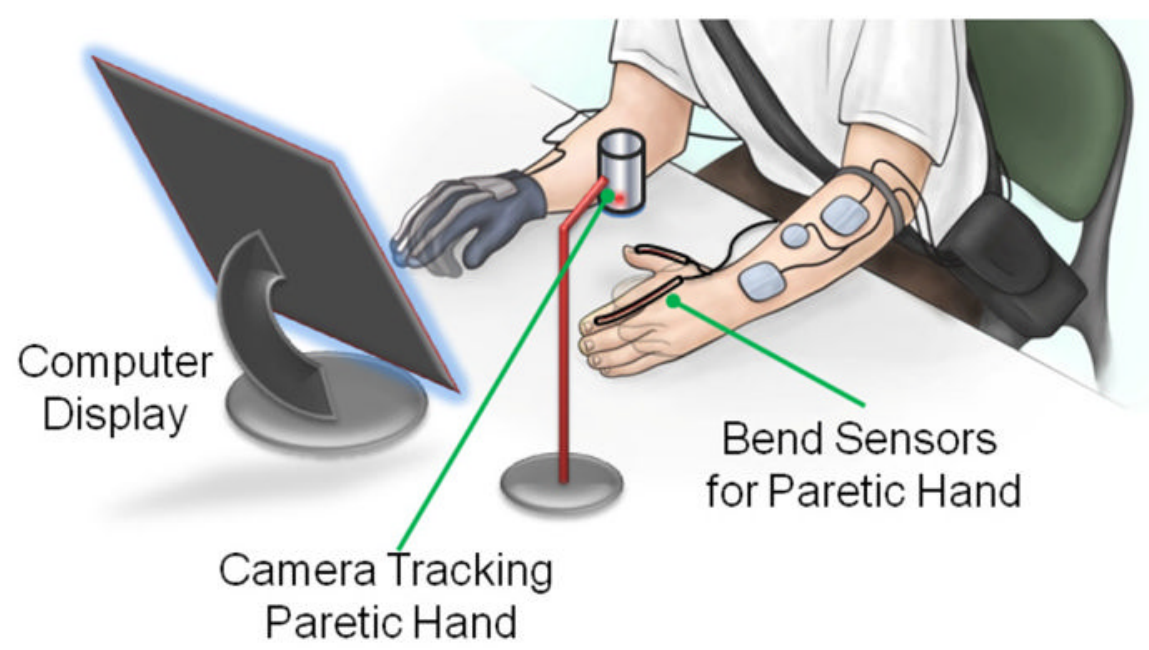

Figure 6.

CCFES+VR system for improving recovery of hand movement and function (C2011 Cleveland FES Center 
TABLE I

Changes from Baseline for Participants in Arm+Hand CCFES Pilot Study

\begin{tabular}{lcc}
\hline \multirow{2}{*}{ Measure } & \multicolumn{2}{c}{ Participant } \\
\cline { 2 - 3 } & $\mathbf{1}$ & $\mathbf{2}$ \\
\hline Fugl-Meyer Score & +7 & +10 \\
\hline Hand Opening $(\mathrm{cm})$ & +0 & +0 \\
\hline Elbow Extension & $+13^{\circ}$ & $+10^{\circ}$ \\
\hline Elbow Tracking Error & $-36 \%$ & $-8 \%$ \\
\hline Elbow Extension during Hand Opening & $+16^{\circ}$ & $+5^{\circ}$ \\
\hline Hand Opening (cm) during Elbow Extension & +0 & +0 \\
\hline
\end{tabular}

